\title{
An Unconstrained Hamiltonian Formulation for Incompressible Fluid Flow
}

\author{
John H. Maddocks, Robert L. Pego \\ Department of Mathematics and Institute for Physical Science and Technology, University of \\ Maryland, College Park, MD 20742, USA
}

Received April 19, 1994

\begin{abstract}
The equations governing the time evolution of an ideal fluid in material coordinates are expressed as an unconstrained canonical Hamiltonian system. The incompressibility of the flow is consequent upon certain first integrals of the motion. The variable conjugate to the configuration field is not the usual linear momentum, but is instead a quantity that is related to linear momentum through an auxiliary scalar field whose time derivative is the pressure. The definition of the Hamiltonian involves a minimization with respect to this auxiliary field. The method of derivation may be generally applied to obtain unconstrained Hamiltonian descriptions of Lagrangian field equations subject to pointwise constraints.
\end{abstract}

\section{Introduction}

In this note we present a novel Hamiltonian formulation of the equations of ideal fluid flow, expressed in material coordinates. The Hamiltonian system is described by Eqs. (6) below, which have the following noteworthy features: First, the variables are unconstrained - incompressibility is not guaranteed from configuration constraints, but rather arises from integrals of the evolution. Offsetting this, the definition of the Hamiltonian involves a minimization with respect to an auxiliary field. An associated feature is that the conjugate momentum has an unobservable component that does not affect the physical flow.

Surveys and extensive references describing prior variational and Hamiltonian formulations of incompressible, inviscid fluid flow are given by Benjamin [2], Holm et al. [9], and Serrin [13], for example. Our description arises from a general approach for constrained systems that is not restricted to problems in fluid mechanics. Rather, it is widely applicable for obtaining unconstrained Hamiltonian dynamical systems from Lagrangian field equations that are subject to pointwise constraints. We first came upon the approach in the context of the dynamics of an inextensible elastic rod [7], where the associated Hamiltonian system facilitated the characterization 
and stability analysis of solitary waves. When restricted to finite dimensional problems (i.e., to problems described by ordinary differential equations), our procedure reduces to a variant of "vakonomic" mechanics [1]. In the language of geometric mechanics, the method starts with Lagrangian dynamics subject to holonomic constraints, and derives a Hamiltonian description defined on the cotangent bundle of the entire ambient configuration space, rather than one restricted to a given level set of the constraints. In the resulting Hamiltonian dynamics, the constraints are recovered as integrals of the motion.

In the new approach, the variable that is conjugate to the configuration variable is neither the classic (linear) momentum nor the impulse. It seems appropriate to give the quantity a name, and the term impetus has been proposed [7]. The usual momentum and velocity are related to the impetus through an auxiliary variable that we call the striction. The striction is a Lagrange multiplier associated with the constraint arising after differentiation with respect to time. Generally, the time derivative of the striction is a familiar physical quantity. In the case of an ideal fluid, the time derivative of the striction is the pressure field.

In our formulation of ideal fluid flow, the Hamiltonian depends only upon the configuration and impetus fields, but evaluation of the Hamiltonian involves minimization over an appropriate class of striction fields. Consequently, the striction satisfies an elliptic boundary value problem whose coefficients depend upon the configuration and impetus. If one takes the material time derivative of this boundary value problem, the elliptic system that determines the pressure is obtained. Moreover, the impetus, striction and velocity are related through a variant of the Helmholtz decomposition. From the point of view of analysis or numerical computation, a possible advantage of our formulation is that the boundary condition for striction at a fixed boundary arises as the natural boundary condition associated with the minimization, and does not involve evaluation of the velocity gradient at the boundary (as required by the corresponding boundary condition for the pressure).

In Sect. 2 below we present our Hamiltonian formulation for the dynamics of an ideal fluid, and in Sect. 3 discuss its derivation. The density may be inhomogeneous (as in a stratified fluid), and both fixed and free boundaries are permitted. Then in Sect. 4 we present two further variants. First we show that with modifications in the initial expression taken for the Lagrangian, our approach generates a family of Hamiltonian descriptions of ideal fluid flow involving different conjugate variables, or impetuses. The version given in Sect. 2 is in some respects the most straightforward, but other systems are of interest. In particular we can recover the Hamiltonian description of fluid flow obtained by Kuz'min [10], Osedelets [12] and Buttke [5] for constant density fluids without free boundaries. The variable corresponding to the choice of impetus made in Sect. 4.1 was called "vortex momentum density" by Kuz'min [10], "velicity" by Buttke [5], and "magnetization variables" by Chorin [6]. Chorin uses these variables to study the statistical mechanics of vortices on lattices. Finally, we close with a description of how the Navier-Stokes equations for homogeneous incompressible flows in domains without fixed boundaries can be written in terms of the impetus variable.

\section{Description}

2.1. Consider an incompressible, inviscid, but possibly inhomogeneous fluid, occupying a region in $R^{N}(N=2$ or 3$)$, that is described by a (smooth) Lagrangian 
flow map $(\alpha, t) \mapsto x$, where $x(\alpha, t) \in R^{N}$ are the current coordinates of the material particle with reference coordinates $\alpha$ lying in a fixed region $\Omega$. The superscript will denote the material time derivative, so that $\dot{x}=\partial x(\alpha, t) / \partial t$ is the velocity. The Jacobian matrix for the coordinate change $\alpha \mapsto x$ is denoted $F=\partial x / \partial \alpha$ (or $\left.F_{i j}=\partial x_{i} / \partial \alpha_{j}\right)$, and $F^{T}$ denotes the transpose. The notation $\nabla=\left(\partial / \partial x_{1}, \ldots, \partial / \partial x_{N}\right)$ is reserved for derivatives in Eulerian coordinates - if $f(\alpha)$ is a function and $v(\alpha)$ a (column) vector field on $\Omega$, then

$$
\nabla f=\left(\frac{\partial f}{\partial \alpha} F^{-1}\right)^{T}, \quad \nabla \cdot v=\operatorname{tr}(\nabla v)=\operatorname{tr}\left(\frac{\partial v}{\partial \alpha} F^{-1}\right), \quad v \cdot \nabla f=\frac{\partial f}{\partial \alpha} F^{-1} v .
$$

The region currently occupied by the fluid is denoted $x(\Omega)$ or $x(\Omega, t)$.

We suppose the fluid boundary (if any) to be in two, smooth, disjoint parts, as for a fluid of finite depth over an infinite bottom. That is, we assume $\partial \Omega=\Gamma_{0} \cup \Gamma_{1}$, where $\Gamma_{0}$ is a free surface on which the pressure $p$ vanishes, and $\Gamma_{1}$ is that part of the fluid in contact with an impenetrable fixed boundary $x\left(\Gamma_{1}\right)$ with outward unit normal $n$. Either or both of the boundaries $\Gamma_{0}$ or $\Gamma_{1}$ could be absent. Thus the boundary conditions are:

$$
p=0 \quad \text { on } \Gamma_{0}, \quad n \cdot \dot{x}=0 \quad \text { on } \Gamma_{1} .
$$

If the domain is unbounded we assume the fluid to be at rest at infinity. We let $\rho=\rho(\alpha)$ denote the Lagrangian fluid density (assumed to be independent of $t$ ). That is, $\rho(\alpha)$ is the mass per unit volume in the reference configuration.

2.2. We first describe the new Hamiltonian formulation, and later derive and motivate it. The dependent variables are the configuration field $x$ and a conjugate variable $y$ that we shall call the impetus. Given fields $(x(\alpha), y(\alpha))$ with $\operatorname{det} \partial x / \partial \alpha>0$, we define the Hamiltonian by

$$
\mathscr{H}(x, y)=\min _{\Lambda} \int_{\Omega} \frac{1}{2} \rho v^{2} d \alpha, \quad \text { subject to } \Lambda=0 \text { on } \Gamma_{0},
$$

where the variable $v(x, y, \Lambda)$ (which turns out to be the velocity) is defined via the equation

$$
\rho v=y-\nabla \Lambda \operatorname{det} \frac{\partial x}{\partial \alpha} .
$$

We call the scalar field $\Lambda=\Lambda(\alpha)$ the striction. The first order conditions associated with the variational principle (3) give rise to a second-order linear elliptic partial differential equation for $\Lambda$. As we shall compute below, this means that $\Lambda$ must solve the boundary value problem

$$
\nabla \cdot v(x, y, \Lambda)=0, \quad n \cdot v=0 \quad \text { on } \Gamma_{1}, \quad \Lambda=0 \quad \text { on } \Gamma_{0} .
$$

The boundary condition that applies on the fixed boundary $\Gamma_{1}$ arises as the natural boundary condition associated with the variational principle (3).

As we verify in Sect. 2.4 below, the canonical Hamiltonian system resulting from (3) is

$$
\dot{x}=\frac{\delta \mathscr{H}}{\delta y}=v, \quad \dot{y}=-\frac{\delta \mathscr{H}}{\delta x}=-(\nabla v)^{T} \nabla \Lambda \operatorname{det} \frac{\partial x}{\partial \alpha},
$$


where $\Lambda(x, y)$ and $v(x, y, \Lambda)$ are determined from Eqs. (4) and (5). Note that the first equation in (6) implies that $v$ is the velocity field.

2.3. We next demonstrate that Eqs. (6) imply Euler's equations for incompressible flow. First, we note that $\operatorname{det} \partial x / \partial \alpha$, evaluated at any fixed material point $\alpha$, is a conserved quantity for the dynamical system (6). Indeed, the matrix $F=\partial x / \partial \alpha$ satisfies the linear differential equation $\dot{F}=(\partial \dot{x} / \partial x)(\partial x / \partial \alpha)=(\nabla v) F$, so by Abel's formula for the determinant, and condition (5),

$$
\frac{\partial}{\partial t}(\operatorname{det} F)=\nabla \cdot v \operatorname{det} F=0 \text {. }
$$

Thus the flow is automatically incompressible, and the assumption $\operatorname{det} F>0$ is a condition on initial data only.

We compute, using (1) and the relation $\partial\left(F^{-1}\right) / \partial t=-F^{-1} \dot{F} F^{-1}$, that

$$
\rho \dot{v}=\frac{\partial}{\partial t}(y-\nabla \Lambda \operatorname{det} F)=\dot{y}-\operatorname{det} F\left(\nabla \dot{\Lambda}-\nabla v^{T} \nabla \Lambda\right)=-\nabla \dot{\Lambda} \operatorname{det} F .
$$

These are Euler's equations with pressure

$$
p=\dot{\Lambda}
$$

and Eulerian density $\bar{\rho}=\rho / \operatorname{det} F$. Moreover, the boundary conditions (2) are satisfied.

It is of interest to note that the minimization in definition (3) implies that $v$ and $\Lambda$ can be determined from $y$ through the decomposition

$$
y=\rho v+\nabla \Lambda \operatorname{det} F, \quad \nabla \cdot v=0,
$$

subject to the boundary conditions

$$
\Lambda=0 \quad \text { on } \Gamma_{0}, \quad n \cdot v=0 \text { on } \Gamma_{1} .
$$

If $\rho / \operatorname{det} F \equiv 1$, then (10) is the usual Helmholtz decomposition.

2.4. So far we have demonstrated that the system (6) is an unconstrained system of evolution equations that generates an incompressible flow which satisfies Euler's equations. However it remains to verify that the system is indeed Hamiltonian, in the sense that the expressions for the variational derivatives appearing in (6) are valid for the Hamiltonian (3).

Regarding $\Lambda$ at first as a field independent of $x$ and $y$, we define a preHamiltonian $\tilde{\mathscr{H}}(x, y, \Lambda)$ by

$$
\tilde{\mathscr{H}}(x, y, \Lambda)=\int_{\Omega} \frac{1}{2} \rho v^{2} d \alpha,
$$

where $v=v(x, y, \Lambda)$ is defined by Eq. (4). The definition (3) of the Hamiltonian is then equivalent to

$$
\mathscr{H}(x, y)=\min _{\Lambda} \tilde{\mathscr{H}}(x, y, \Lambda),
$$

subject to the boundary condition $\Lambda=0$ on $\Gamma_{0}$. It is evident that $\tilde{\mathscr{H}}$ is convex in $\Lambda$ provided $\operatorname{det} F>0$, so the minimum will exist in an appropriate function space. 
The fields $x$ and $y$ uniquely determine the minimizing field $\Lambda=\Lambda(x, y)$, and for this $\Lambda$ we have $\delta \tilde{\mathscr{H}} / \delta \Lambda=0$. This identity means that the variational derivatives of $\mathscr{H}$ may be computed directly from the relations

$$
\frac{\delta \mathscr{H}}{\delta x}(x, y)=\frac{\delta \tilde{\mathscr{H}}}{\delta x}(x, y, \Lambda), \quad \frac{\delta \mathscr{H}}{\delta y}(x, y)=\frac{\delta \tilde{\mathscr{H}}}{\delta y}(x, y, \Lambda) .
$$

It is straightforward to compute that $\delta \mathscr{H} / \delta y=v$. Then the first of Hamilton's equations in (6) reduces to the statement that the variable $v$ is the velocity field. Concomitantly the Hamiltonian (3) can be seen to be the kinetic energy (integrated over the Lagrangian domain).

We next demonstrate that the incompressibility condition (5) is equivalent to the condition $\delta \tilde{\mathscr{H}} / \delta \Lambda=0$. With $\hat{\Lambda}$ denoting the variation in $\Lambda$, we find

$$
\left.\frac{d}{d \varepsilon} \tilde{\mathscr{H}}(x, y, \Lambda+\varepsilon \hat{\Lambda})\right|_{\varepsilon=0}=-\int_{\Omega} v^{T} \nabla \hat{\Lambda} \operatorname{det} F d \alpha .
$$

Integrate by parts after changing to Eulerian variables, then change back to material coordinates. One finds that the expression above equals

$$
\int_{\Omega} \hat{\Lambda}(\nabla \cdot v) \operatorname{det} F d \alpha-\int_{o x(\Omega)} \hat{\Lambda}(n \cdot v) d S_{x},
$$

from which the desired equivalence follows.

Finally we compute the $x$-variation of $\tilde{\mathscr{H}}$ due to a perturbation $\hat{x}(\alpha)$. Note that

$$
\frac{\delta}{\delta x}(\operatorname{det} F) \hat{x}=\left.\frac{d}{d \varepsilon} \operatorname{det} \frac{\partial(x+\varepsilon \hat{x})}{\partial \alpha}\right|_{\varepsilon=0}=\operatorname{tr}\left(\frac{\partial \hat{x}}{\partial \alpha} F^{-1}\right) \operatorname{det} F=\nabla \cdot \hat{x} \operatorname{det} F .
$$

Then we obtain

$$
\begin{aligned}
\int_{\Omega} \frac{\delta \tilde{\mathscr{H}}}{\delta x} \hat{x} d \alpha= & \int_{\Omega}(\nabla \Lambda)^{T}(\nabla \hat{x}-(\nabla \cdot \hat{x}) I) v \operatorname{det} F d \alpha \\
= & \int_{\partial x(\Omega)}(\hat{x} \cdot \nabla \Lambda)(n \cdot v)-(n \cdot \hat{x})(v \cdot \nabla \Lambda) d S_{x} \\
& +\int_{x(\Omega)} \nabla \Lambda^{T}(\nabla v-(\nabla \cdot v) I) \hat{x} d x .
\end{aligned}
$$

The boundary term vanishes because $\nabla \Lambda$ is normal to the free surface $\Gamma_{0}$, and variations satisfy $n \cdot \hat{x}=0$ on the fixed boundary $\Gamma_{1}$. Since $\nabla \cdot v=0$ by (5), upon returning to material coordinates we can recover the second of Eqs. (6), so that the system is indeed Hamiltonian, as previously claimed.

2.5. The Hamiltonian defined in (3) admits a symmetry (or gauge freedom) associated with certain translations of the impetus $y$. The symmetry leaves the flow map $x(\alpha, t)$ unchanged. Explicitly, whenever $(x, y)$ is a solution of $(6)$, and $\tilde{\Lambda}(\alpha)$ is any given smooth function with $\tilde{\Lambda}=0$ on $\Gamma_{0}$, then the pair $(x, y+\nabla \tilde{A} \operatorname{det} F)$ is another solution of (6). This is the sense in which part of the impetus $y$ is unobservable the initial values of the impetus $y$ may be altered by a term of the form $\nabla \tilde{\Lambda} \operatorname{det} F$ with no effect on the flow map $x(\alpha, t)$. 
The symmetry of the Hamiltonian is associated with the conserved quantities

$$
\mathscr{C}=\int_{\Omega} \tilde{\Lambda}(\alpha) \operatorname{det} F d \alpha,
$$

for it may be verified that

$$
\frac{\delta \mathscr{C}}{\delta y}=0, \quad-\frac{\delta \mathscr{C}}{\delta x}=\nabla \tilde{\Lambda} \operatorname{det} F
$$

The arbitrariness of $\tilde{\Lambda}(\alpha)$ in (15) then implies the pointwise incompressibility condition, namely that $\operatorname{det} F$ is independent of time.

While the Hamiltonian can be seen to be a convex function of the impetus variable $y$, its invariance to certain translations implies an absence of strict convexity. The pre-Hamiltonian defined through (12) and (4) is a strictly convex function of the impetus, but the minimization in the definition (13) of the Hamiltonian generates the translational invariance.

\section{Derivation}

It remains to motivate our choice of impetus as the conjugate variable leading to the new Hamiltonian system. In point of fact, the Hamiltonian formulation we have described can be found from a constrained Lagrangian action principle via a Legendre transform, in an almost classic construction. The ideas that we describe carry over to Lagrangians that are convex in the velocity, and a wide class of holonomic pointwise constraints. But for the sake of definiteness, we here restrict attention to the case of an incompressible fluid.

3.1. The framework is as follows. Let

$$
L(x, \dot{x})=\int_{\Omega} \frac{1}{2} \rho \dot{x}^{2} d \alpha,
$$

be the Lagrangian. To incorporate the incompressibility constraint $\operatorname{det} F=$ const, we first differentiate in time, writing instead $\operatorname{tr}\left((\partial \dot{x} / \partial \alpha) F^{-1}\right) \operatorname{det} F=0$, or $(\nabla \cdot \dot{x}) \operatorname{det} F=0$. Then the full variational Lagrangian incorporates the differentiated constraint with a multiplier, or striction, field $\Lambda(\alpha, t)$ :

$$
\mathscr{L}(x, \dot{x}, \Lambda)=\int_{\Omega} \frac{1}{2} \rho \dot{x}^{2}-\Lambda(\nabla \cdot \dot{x}) \operatorname{det} F d \alpha .
$$

According to the usual (Lagrangian) action principle, the appropriate equations of motion are the Euler-Lagrange equations $-(\partial / \partial t)(\delta \mathscr{L} / \delta \dot{x})+\delta \mathscr{L} / \delta x=0$, supplemented with the constraint $\delta \mathscr{L} / \delta \Lambda=0$, and the boundary conditions (2). With calculations analogous to the ones described above, one may easily compute $\delta \mathscr{L} / \delta \dot{x}$. Then the impetus $y$ is defined by

$$
y \equiv \frac{\delta \mathscr{L}}{\delta \dot{x}}=\rho \dot{x}+\nabla \Lambda \operatorname{det} F
$$

This equation is then inverted to solve for $\dot{x}=v(x, y, \Lambda)=\rho^{-1}(y-\nabla \Lambda \operatorname{det} F)$, and the pre-Hamiltonian is defined through the Legendre transformation

$$
\tilde{\mathscr{H}}(x, y, \Lambda)=\int_{\Omega} y^{T} v d \alpha-\mathscr{L}(x, v, \Lambda)=\int_{\Omega} \frac{1}{2} \rho v^{2} d \alpha .
$$


The last identity uses an integration by parts and the boundary conditions $\Lambda=0$ on $\Gamma_{0}, n \cdot v=0$ on $\Gamma_{1}$. The constraint $\delta \mathscr{L} / \delta \Lambda=0$ then yields (5), which determines $\Lambda$ in terms of $(x, y)$. Since (5) is equivalent to $\delta \tilde{\mathscr{H}} / \delta \Lambda=0$, and the pre-Hamiltonian $\tilde{\mathscr{H}}$ is a convex function of $\Lambda$, the Hamiltonian may accordingly be defined via minimization as in (3), leading to the Hamiltonian system (6).

3.2. The algorithm to derive the Hamiltonian for the impetus formulation of the dynamics can be described in another equivalent way, which proves slightly more convenient in many examples. From the Lagrangian (17) we obtain the momentum density

$$
\xi=\frac{\delta L}{\delta \dot{x}}=\rho \dot{x},
$$

and the associated Hamiltonian defined through the classic Legendre transform, namely

$$
H(x, \xi)=\frac{1}{2} \int_{\Omega} \frac{\xi^{2}}{\rho} d \alpha .
$$

Then the impetus is defined through (19) as before, which can be re-written

$$
\xi=y-\nabla \Lambda \operatorname{det} F
$$

With this relation, the pre-Hamiltonian (20) has the equivalent definition

$$
\tilde{\mathscr{H}}(x, y, \Lambda)=H(x, \xi) \text {. }
$$

3.3. The only direct antecedent of the approach described above that is known to us is the theory of vakonomic mechanics [1]. That body of work is concerned with systems described by ordinary differential equations, and the primary focus is on issues arising for non-holonomic constraints that do not concern us. Nevertheless, the finite dimensional analogue of the unconstrained impetus variable in Eq. (10) certainly appears in that theory. Arnold et al. [1] also mention that for holonomic constraints, the theory yields Hamilton's equations in "redundant coordinates." However, the formulation utilizing a minimization principle, as in (3), is apparently new, even in the case of ordinary differential equations.

The impetus-striction formulation has certain features in common with two other works. First, Dirac's theory of constraints [8] concerns the construction of a Hamiltonian formulation for Lagrangian dynamics where the Lagrangian is a convex, but not strictly convex, function of the velocities. The Hamiltonian that Dirac constructs is a strictly convex function of the conjugate momenta, but is constrained. The impetus-striction formulation is, at least in a formal sense, dual to Dirac's construction. We start from a constrained Lagrangian that is strictly convex in the velocities, and obtain an unconstrained Hamiltonian that is convex, but not strictly convex, in the impetus variable.

Second, Benjamin and Bridges [3] have also exploited a Hamiltonian system that is only defined after minimization with respect to an auxiliary variable. Their work is in the context of two-fluid flows. They assume irrotational motion in each component, and introduce two velocity potentials. The auxiliary minimization is then used to enforce the appropriate interface conditions at the boundary between the two fluids. 
3.4. The motivation for the choice of impetus variable given in Sec. 3.1 was in terms of the Lagrangian form of the action principle. Consideration of the Hamiltonian form of the action principle provides yet another perspective. The classic constrained action involves the configuration $x$, momentum $\xi$, the Hamiltonian (21) and the pressure $p$ as a Lagrange multiplier:

$$
\int_{t_{0}}^{t_{1}} H(x, \xi)-\int_{\Omega} \xi \cdot \dot{x}+p(\operatorname{det} F-1) d \alpha d t .
$$

Using (14) the Euler-Lagrange equations of (24) with respect to $x, \xi$ and $p$ may be written in the form

$$
\left(\begin{array}{ccc}
0 & I & 0 \\
-I & 0 & 0 \\
0 & 0 & 0
\end{array}\right)\left(\begin{array}{l}
x \\
\xi \\
p
\end{array}\right)_{t}+\left(\begin{array}{c}
\operatorname{det} F \nabla p \\
\xi / \rho \\
1-\operatorname{det} F
\end{array}\right)=\left(\begin{array}{l}
0 \\
0 \\
0
\end{array}\right)
$$

which can be recognized as a degenerate symplectic system. After we substitute $\dot{A}=p$ and integrate by parts with respect to both space and time, the action (24) takes the form

$$
\int_{t_{0}}^{t_{1}} H(x, \xi)-\int_{\Omega}(\xi+\operatorname{det} F \nabla \Lambda) \cdot \dot{x} d \alpha d t .
$$

Now eliminate the momentum $\xi$ in favor of the impetus $y=(\xi+\operatorname{det} F \nabla \Lambda)$ to obtain the action in the form

$$
\int_{t_{0}}^{t_{1}} H(x, y-\operatorname{det} F \nabla \Lambda)-\int_{\Omega} y \cdot \dot{x} d \alpha d t .
$$

The Euler-Lagrange equations of (27) with respect to $x, y$ and $\Lambda$ are:

$$
\left(\begin{array}{ccc}
0 & I & 0 \\
-I & 0 & 0 \\
0 & 0 & 0
\end{array}\right)\left(\begin{array}{l}
x \\
y \\
\Lambda
\end{array}\right)_{t}+\left(\begin{array}{c}
\delta \tilde{\mathscr{H}} / \delta x \\
\delta \tilde{\mathscr{H}} / \delta y \\
\delta \tilde{\mathscr{H}} / \delta \Lambda
\end{array}\right)=\left(\begin{array}{l}
0 \\
0 \\
0
\end{array}\right)
$$

where $\tilde{\mathscr{H}}(x, y, \Lambda)$ is the pre-Hamiltonian defined in (22) and (23). Notice that in each of the degenerate symplectic systems (25) and (28) the third equation enforces the incompressibility constraint. However, pointwise minimization of the action (27) with respect to the striction $\Lambda$ implies the third equation in the system (28), and yields the classic action

$$
\int_{t_{0}}^{t_{1}} \mathscr{H}(x, y)-\int_{\Omega} y \cdot \dot{x} d \alpha d t
$$

according to the definition of $\mathscr{H}$ in (13). The Euler-Lagrange equations of (29) are

$$
\left(\begin{array}{cc}
0 & I \\
-I & 0
\end{array}\right)\left(\begin{array}{l}
x \\
y
\end{array}\right)_{t}+\left(\begin{array}{c}
\delta \mathscr{H} / \delta x \\
\delta \mathscr{H} / \delta y
\end{array}\right)=\left(\begin{array}{l}
0 \\
0
\end{array}\right)
$$

which is a non-degenerate symplectic system equivalent to the evolution equations in $(6)$. 


\section{Variants}

The procedure we have described can be modified in various ways. For example, heretofore the Hamiltonian $\mathscr{H}$ has been the total kinetic energy, but it is straightforward to include a potential energy term of the form $\int_{\Omega} V(x, \alpha) d \alpha$, in which case the Hamiltonian is the total energy. However we here confine ourselves to a description of two other variants.

4.1. First, in the absence of free boundaries, the "vortex momentum density" or "velicity" formulation of incompressible inviscid fluid flow $[5,10,12]$ can be recovered and extended to variable density flows. Instead of (18), one begins with a different Lagrangian, namely the kinetic energy written in an Eulerian description:

$$
L(x, \dot{x})=\int_{x(\Omega)} \frac{1}{2} \bar{\rho} \dot{x}^{2} d x=\int_{\Omega} \frac{1}{2} \bar{\rho} \dot{x}^{2} \operatorname{det} F d \alpha .
$$

In this formulation $\bar{\rho}(\alpha)=\rho(\alpha) /|F(\alpha, 0)|$. That is, $\bar{\rho}(\alpha)$ is the mass per unit volume in the initial configuration. In particular it is important to realise that, a priori, the term $\operatorname{det} F$ appearing in (31) could be a function of time.

Because the Lagrangian is changed, so also are the definitions of the impetus and striction variables, and here they will be denoted $z$ and $\Theta$. As above one computes

$$
\begin{gathered}
z \equiv \frac{\delta \mathscr{L}}{\delta \dot{x}}=(\bar{\rho} \dot{x}+\nabla \Theta) \operatorname{det} F, \quad \bar{\rho} v=z \operatorname{det} F^{-1}-\nabla \Theta \\
\tilde{\mathscr{H}}(x, z, \Theta)=\int_{\Omega} z^{T} v d \alpha-\mathscr{L}=\int_{\Omega} \frac{1}{2} \bar{\rho} v^{2} \operatorname{det} F d \alpha .
\end{gathered}
$$

Then with $\mathscr{H}=\min _{\Theta} \tilde{\mathscr{H}}(x, z, \Theta)($ which implies $\nabla \cdot v(x, z, \Theta)=0)$, the canonical Hamiltonian equations are

$$
\dot{x}=v, \quad \dot{z}=-\left((\nabla v)^{T} \nabla \Theta+\nabla\left(\frac{1}{2} \bar{\rho} v^{2}\right)\right) \operatorname{det} F .
$$

From these follows $\partial(\operatorname{det} F) / \partial t=0$ and

$$
\bar{\rho} \dot{v}=\frac{\partial}{\partial t}\left(z \operatorname{det} F^{-1}-\nabla \Theta\right)=\dot{z} \operatorname{det} F^{-1}-\nabla \dot{\Theta}+\nabla v^{T} \nabla \Theta=-\nabla\left(\dot{\Theta}+\frac{1}{2} \bar{\rho} v^{2}\right),
$$

which are Euler's equations with pressure

$$
p=\dot{\Theta}+\frac{1}{2} \bar{\rho} v^{2} .
$$

The difficulty with free boundaries is revealed by the expression (33) relating the pressure to the striction, for in contrast to the analogous identity (9) arising in Sect. 2.3, here the free surface condition $p=0$ cannot be expressed as a simple boundary condition on the striction $\Theta$.

In the absence of free boundaries Buttke [5] refers to any variable $y / \rho$ found from a Helmholtz decomposition of the form (10) (in the case $\operatorname{det} F=1$ and $\rho$ constant in space) as a "velicity," and describes the dynamics for the particular velicity $M=z / \rho$, where $z$ is the impetus associated with the Lagrangian (31). In connection with his numerical work on unbounded domains, Buttke [5] asserts that the gauge freedom described in Sect. 2.5 can be exploited to guarantee that the particular impetus $z$ (or equivalently the velicity $M$ ) has compact support for all time. 
One might incorporate $\operatorname{det} F$ into the Lagrangian in a rather more general way than is taken in either (17) or (31). If the kinetic energy is originally written in the form

$$
L=\int_{\Omega} \frac{1}{2} \bar{\rho} \dot{x}^{2} g_{1}(\operatorname{det} F) d \alpha,
$$

with $\bar{\rho}=\rho / g_{1}(\operatorname{det} F(\alpha, 0))$, and the constraint is written $g_{2}(\operatorname{det} F)=0$, then one obtains the modified Lagrangian

$$
\mathscr{L}=\int_{\Omega} \frac{1}{2} \bar{\rho} v^{2} g_{1}-\left(\Lambda g_{2}^{\prime}\right)(\nabla \cdot \dot{x}) \operatorname{det} F d \alpha .
$$

The impetus variable is then $y=\bar{\rho} v g_{1}+\nabla\left(\Lambda g_{2}^{\prime}\right) \operatorname{det} F$, the Hamiltonian is the kinetic energy, and the associated dynamical system is

$$
\dot{x}=v, \quad \dot{y}=-\left[(\nabla v)^{T} \nabla\left(\Lambda g_{2}^{\prime}\right)+\nabla\left(\frac{1}{2} \bar{\rho} v^{2} g_{1}^{\prime}\right)\right] \operatorname{det} F .
$$

Consequently this generalization yields a family of systems, but does not yield any essentially new structure. In some sense the functions $g_{1}(s) \equiv 1$ and $g_{2}(s) \equiv s-1$ that lead to our system (6) are the simplest choices.

4.2. Finally, we show how to modify the Hamiltonian formulation (6) of ideal flow to include a viscosity term, yielding the incompressible Navier-Stokes equations. It is simplest to do this in the case of constant density, $\rho \equiv 1 \equiv \operatorname{det} F$, and in the complete absence of boundaries, so $\Omega=R^{N}$. (Such assumptions were also made by Osedelets [12] and Buttke [5].)

It then suffices to consider the system

$$
\dot{x}=v, \quad \dot{y}=-(\nabla v)^{T} \nabla \Lambda+v \Delta v .
$$

Here $v$ and $\Lambda$ are determined by the Helmholtz decomposition $y=v+\nabla \Lambda$ with $\nabla \cdot v=0$ as in (10), $\Delta$ is the Eulerian Laplacian, and $v$ is the viscosity coefficient. The incompressibility condition $\partial(\operatorname{det} F) / \partial t=0$, and the relation $\dot{x}=v$ involve only kinematics and therefore follow just as was described in Sect. 2.3 and Sect. 2.4. The additional term in the second of Eqs. (34) is merely the appropriate viscous stress arising in the force balance. The system (34) is of the general form of a dissipatively perturbed Hamiltonian system in which the structure matrix has been modified by the addition of a negative semi-definite symmetric operator:

$$
\left(\begin{array}{l}
x \\
y
\end{array}\right)_{t}=\left(\begin{array}{cc}
0 & I \\
-I & \nu \Delta
\end{array}\right)\left(\begin{array}{c}
\delta \mathscr{H} / \delta x \\
\delta \mathscr{H} / \delta y
\end{array}\right) .
$$

Such systems can be convenient for stability analyses. (See, for example, Maddocks \& Overton [11] or Bloch et al. [4].) However in the system (34) the term $v \Delta v$ is a degenerate operator on the impetus $y$. Consequently it is of interest to note that the system

$$
\dot{x}=v, \quad \dot{y}^{*}=-(\nabla v)^{T} \nabla \Lambda^{*}+v \Delta y^{*}
$$

is also equivalent to the incompressible Navier-Stokes equations. Here the variable $y^{*}$ may evolve differently from $y$. The first equation and incompressibility condition 
again involve only kinematic considerations. Moreover by (36) and the decomposition $y^{*}=v+\nabla \Lambda^{*}$,

$$
\begin{aligned}
\dot{v}=\frac{\partial}{\partial t}\left(y^{*}-\nabla \Lambda^{*}\right) & =\dot{y}^{*}-\nabla \dot{\Lambda}^{*}+(\nabla v)^{T} \nabla \Lambda^{*} \\
& =-\nabla \dot{\Lambda}^{*}+v \Delta\left(v+\nabla \Lambda^{*}\right) \\
& =-\nabla\left(\dot{\Lambda}^{*}-v \Delta \Lambda^{*}\right)+v \Delta v,
\end{aligned}
$$

which are the Navier-Stokes equations, with the pressure related to the striction by $\dot{\Lambda}^{*}=v \Delta \Lambda^{*}+p$. Notice that Eqs. (34) and (36) generate different time evolutions for the variables $y$ and $y^{*}$. But the velocity fields $v(\alpha, t)$ and flow maps $x(\alpha, t)$ for the two systems coincide.

Acknowledgement. It is a pleasure to thank Professors R. E. Caflisch and P. J. Olver and Drs. T. Bridges and P. Smereka for their detailed comments on early versions of this note. The research of JHM was supported by the AFOSR and ONR. The research of RLP was supported by the NSF.

\section{References}

1. Arnold, V.I., Kozlov, V.V., Neishtadt, A.I.: Mathematical aspects of classical and celestial mechanics. In: Arnold, V.I. (ed.) Dynamical Systems III, Encyclopaedia of Mathematical Sciences, vol. 3. Berlin, Heidelberg, New York: Springer-Verlag, 1988 (See pp. 31-38)

2. Benjamin, T.B.: Impulse, flow force and variational principles. IMA J. Appl. Math. 32, 3-68 (1984)

3. Benjamin, T.B., Bridges, T.J.: Reappraisal of the Kelvin-Helmholtz problem. Part 1, Hamiltonian structure. Preprint 1993

4. Bloch, A., Krishnaprasad, P.S., Marsden, J.E., Ratiu, T.S.: Dissipation induced instabilities. Ann. Inst. H. Poincaré, Anal. Non Linéaire 11, 1-54 (1994)

5. Buttke, T.F.: Velicity methods: Lagrangian numerical methods which preserve the Hamiltonian structure of incompressible fluid flow. In: Beale, J.T., Cottet, G.H., Huberson, S. (eds.) Vortex Flows and Related Numerical Methods. NATO Adv. Sci. Inst. Ser. C Math. Phys. Sci., vol. 395, pp. 39-57. Dordrecht, Boston, London: Kluwer, 1993

6. Chorin, A.: Vortex Phase Transitions in $2 \frac{1}{2}$ dimensions. Lawrence Berekeley Laboratory Report LBL-34769 (1993)

7. Dichmann, D.J., Maddocks, J.H., Pego, R.L.: Hamiltonian dynamics of an elastica and the stability of solitary waves. Arch. Rat. Mech. Anal. (submitted)

8. Dirac, P.A.M.: On generalized Hamiltonian dynamics. Can. J. Math. 2, 129-148 (1950)

9. Holm, D.D., Marsden, J.E., Ratiu, T.S.: Hamiltonian structure of continuum mechanics in material, inverse material, spatial and convective representations. In: Hamiltonian structure and Lyapunov stability for ideal continuum mechanics. Sem. Math. Sup. vol. 100. University of Montreal Press, 1986

10. Kuz'min, G.A.: Ideal incompressible hydrodynamics in terms of the vortex momentum density. Phys. Lett. 96A, 88-90 (1983)

11. Maddocks, J.H., \& Overton, M.L.: Stability theory for dissipatively perturbed Hamiltonian systems. Comm. Pure Appl. Math. (to appear)

12. Osedelets, V.I.: On a new way of writing the Navier-Stokes equation. The Hamiltonian formalism. Comm. Moscow Math. Soc. (1988) [transl.: Russ. Math. Surveys 44, 210-211 (1989)]

13. Serrin, J.: Mathematical principles of classical fluid mechanics. In: Handbuch der Physik, Vol. VIII/1. Berlin, Heidelberg, New York: Springer-Verlag, 1959, pp. 125-263. 
